\title{
Electrostatic potential mapping at ferroelectric domain walls by low- temperature photoemission electron microscopy
}

Cite as: Appl. Phys. Lett. 115, 122903 (2019); https://doi.org/10.1063/1.5117881

Submitted: 02 July 2019 . Accepted: 31 August 2019 . Published Online: 19 September 2019

J. Schaab, K. Shapovalov, P. Schoenherr, J. Hackl, M. I. Khan, M. Hentschel, Z. Yan (D), E. Bourret (D, C. M. Schneider (D), S. Nemsák (D), M. Stengel, A. Cano (D), and D. Meier (iD)
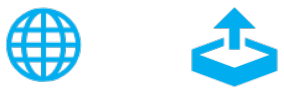

\section{ARTICLES YOU MAY BE INTERESTED IN}

FIB lift-out of conducting ferroelectric domain walls in hexagonal manganites

Applied Physics Letters 115, 122901 (2019); https://doi.org/10.1063/1.5115465

Atomically resolved domain boundary structure in lead zirconate-based antiferroelectrics Applied Physics Letters 115, 122902 (2019); https://doi.org/10.1063/1.5115039

Imaging and characterization of conducting ferroelectric domain walls by photoemission electron microscopy

Applied Physics Letters 104, 232904 (2014); https://doi.org/10.1063/1.4879260

Lock-in Amplifiers Find out more today

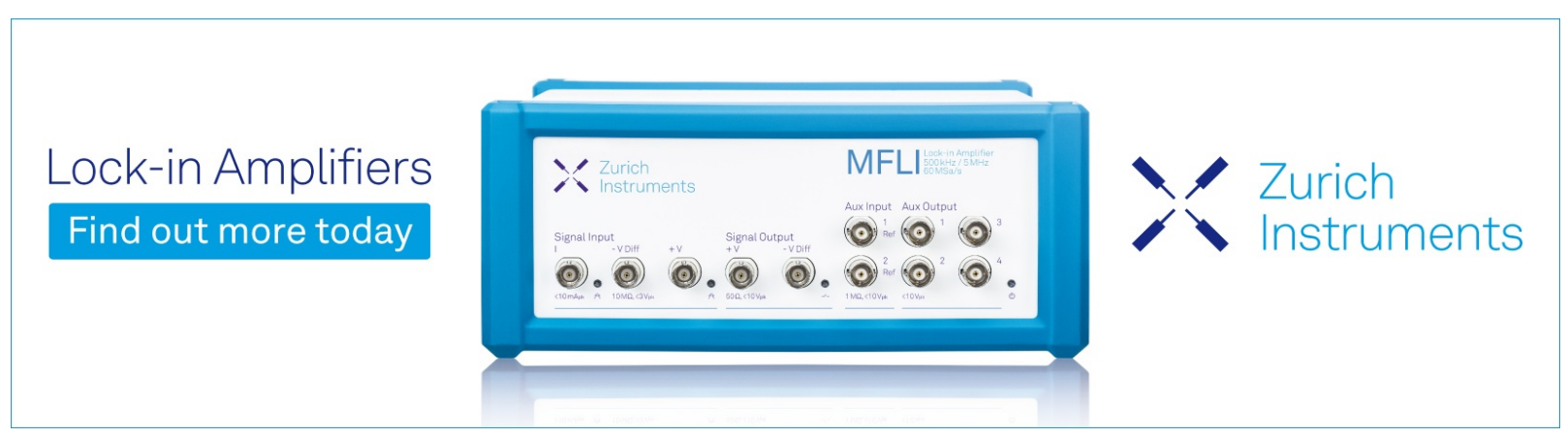




\title{
Electrostatic potential mapping at ferroelectric domain walls by low-temperature photoemission electron microscopy
}

Cite as: Appl. Phys. Lett. 115, 122903 (2019); doi: 10.1063/1.5117881

Submitted: 2 July 2019 - Accepted: 31 August 2019 .

Published Online: 19 September 2019

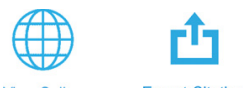

View Online Export Citatior

J. Schaab, ${ }^{7}$ K. Shapovalov, ${ }^{2}$ P. Schoenherr, ${ }^{7}$ J. Hackl, ${ }^{3}$ M. I. Khan, ${ }^{3}$ M. Hentschel, ${ }^{4}$ Z. Yan, ${ }^{5,6}$ (DD E. Bourret, ${ }^{6}$ (D) C. M. Schneider, ${ }^{3,7}$ (D) S. Nemsák, ${ }^{3,8}$ (iD M. Stengel, ${ }^{2,9}$ A. Cano, $^{10}$ (iD and D. Meier ${ }^{1,11, a)}$ (iD)

\author{
AFFILIATIONS \\ 'Department of Materials, ETH Zurich, Vladimir-Prelog-Weg 4, 8093 Zurich, Switzerland \\ ${ }^{2}$ Institut de Ciència de Materials de Barcelona (ICMAB-CSIC), Campus UAB, 08193 Bellaterra, Spain \\ ${ }^{3}$ Forschungszentrum Jülich Peter Grünberg Institute (PGI-6), Leo-Brandt-Strasse, 52425 Jülich, Germany \\ ${ }^{4} 4$ th Physics Institute and Research Center SCoPE, University of Stuttgart, Pfaffenwaldring 57, 70569 Stuttgart, Germany \\ ${ }^{5}$ Department of Physics, ETH Zurich, Otto-Stern-Weg 1, 8093 Zurich, Switzerland \\ ${ }^{6}$ Materials Science Division, Lawrence Berkeley National Laboratory, Berkeley, California 94720, USA \\ ${ }^{7}$ Physics Department, UC Davis, Davis, California 95616, USA \\ ${ }^{8}$ Advanced Light Source, Lawrence Berkeley National Laboratory, 1 Cyclotron Rd, Berkeley, California, 94720, USA \\ ${ }^{9}$ ICREA-Instució Catalana de Recerca i Estudis Avançats, 08010 Barcelona, Spain \\ ${ }^{10}$ Institut Néel, CNRS \& University Grenoble Alpes, 38042 Grenoble, France \\ ${ }^{17}$ Department of Materials Science and Engineering, Norwegian University of Science and Technology, NTNU, \\ 7043 Trondheim, Norway
}

a) Author to whom correspondence should be addressed: dennis.meier@ntnu.no

\begin{abstract}
Low-temperature X-ray photoemission electron microscopy (X-PEEM) is used to measure the electric potential at domain walls in improper ferroelectric $\mathrm{Er}_{0.99} \mathrm{Ca}_{0.01} \mathrm{MnO}_{3}$. By combining X-PEEM with scanning probe microscopy and theory, we develop a model that relates the detected X-PEEM contrast to the emergence of uncompensated bound charges, explaining the image formation based on intrinsic electronic domain-wall properties. In contrast to previously applied low-temperature electrostatic force microscopy (EFM), X-PEEM readily distinguishes between positive and negative bound charges at domain walls. Our study introduces an X-PEEM-based approach for lowtemperature electrostatic potential mapping, facilitating nanoscale spatial resolution and data acquisition times on the order of $0.1-1 \mathrm{~s}$.
\end{abstract}

Published under license by AIP Publishing. https://doi.org/10.1063/1.5117881

Fueled by the idea of domain-wall-based nanoelectronics, ${ }^{1-6}$ tremendous progress has been made over the last decade in measuring the nanoscale physical properties at ferroelectric domain walls. ${ }^{7,8}$ Particularly interesting are so-called charged domain walls in ferroelectrics that, depending on their charge state (positive or negative) and the applied electric field, can be either insulating or conducting compared to the surrounding bulk. ${ }^{16}$ This foreshadows the possibility to create, e.g., atomic scale wires, switches, gates, and transistors. ${ }^{4,9,10}$ The progress in the field is enabled by the continuous advancement of established high-resolution imaging techniques, as well as the development of new microscopy approaches. Modern scanning transmission electron microscopes, for example, allow us to measure the atomic and chemical structures at domain walls with atomic scale precision. ${ }^{9}$ Scanning probe and scanning electron microscopy (SPM and SEM) has become mainstream techniques for accessing domain-wall related transport phenomena, ${ }^{11-18}$ electrostatics, ${ }^{19,20}$ and magnetism. ${ }^{21}$ More recently, X-ray photoemission electron microscopy (X-PEEM) and low energy electron microscopy (LEEM) have been employed to study emergent domain wall physics with a nanoscale spatial resolution. ${ }^{22-24}$

Despite the growing diversity of microscopy methods, the more fundamental investigation of domain walls at low temperatures remains surprisingly difficult. Pioneering studies mostly rely on low-temperature 
SPM, which enabled the observation of conductance at domain walls in $\mathrm{Pb}(\mathrm{Zr}, \mathrm{Ti}) \mathrm{O}_{3}{ }^{25}$ and $\mathrm{Nd}_{2} \mathrm{Ir}_{2} \mathrm{O}_{7}{ }^{26}$ as well as domain-wall magnetism ${ }^{21}$ and uncompensated bound charges ${ }^{20}$ in $\mathrm{Er}_{0.99} \mathrm{Ca}_{0.01} \mathrm{MnO}_{3}$. However, in order to go beyond these SPM-based breakthroughs, expand the currently accessible parameter space, and gain additional insight into the domain-wall nanoscale physics at cryogenic temperature, the introduction of additional low-temperature microscopy approaches is highly desirable.

Here, we demonstrate low-temperature X-PEEM as a powerful imaging and characterization tool for ferroelectric domain walls, providing so far inaccessible information about their electrostatic properties. Combining X-PEEM and SPM, we show that distinct X-PEEM contrasts arise at low temperature at positively (head-to-head) and negatively (tail-to-tail) charged domain walls in the improper ferroelectric $\mathrm{Er}_{0.99} \mathrm{Ca}_{0.01} \mathrm{MnO}_{3}$, ${ }^{19,27}$ which we attribute to uncompensated polarization charges. In comparison to previous EFM studies, ${ }^{20}$ the X-PEEM experiment has the advantages of distinguishing emergent positive and negative potentials and, hence, unambiguously identifying head-to-head and tail-to-tail walls without the need for supplementary piezoresponse force microscopy (PFM) measurements. Furthermore, data acquisition times are on the order of $0.1-1 \mathrm{~s}$, i.e., more than 100 times faster than the line-by-line image formation in EFM.

For our low-temperature X-PEEM experiments, high-quality $\mathrm{ErMnO}_{3}$ and $\mathrm{Er}_{0.99} \mathrm{Ca}_{0.01} \mathrm{MnO}_{3}$ single crystals are grown by the pressurized floating-zone method, ${ }^{28}$ oriented by Laue diffraction, and cut into platelets with the spontaneous polarization $(\mathrm{P} \|[001])$ lying in the surface plane. The samples have a lateral dimension of about $4 \mathrm{~mm}$ and a thickness of $1 \mathrm{~mm}$. To minimize topographical artifacts, the surface is chemo-mechanical polished, leading to a roughness of about $2 \mathrm{~nm}$ (RMS).

Figures 1(a) and 1(b) show PFM images (in-plane contrast) of the ferroelectric domains in $\mathrm{ErMnO}_{3}$ and $\mathrm{Er}_{0.99} \mathrm{Ca}_{0.01} \mathrm{MnO}_{3}$, respectively. Both samples exhibit the sixfold distribution of $+\mathrm{P}$ (dark) and -P (bright) domains characteristic of hexagonal manganites. Room-temperature X-PEEM images, gained on the same samples, are presented in Figs. 1(c) and 1(d). The data in Fig. 1(c) is obtained at a photon energy of $641.5 \mathrm{eV}\left(\mathrm{Mn} \mathrm{L}_{3}\right.$ edge), showing several pronounced bright lines. The lines correspond to conducting tail-to-tail walls in $\mathrm{ErMnO}_{3}$. These walls become visible in $\mathrm{X}$-PEEM due to photoinduced charging as explained in detail in Ref. 22. The photoinduced charging is less pronounced at conducting tail-to-tail walls than for the insulating bulk and headto-head walls. As a consequence, photoexcited electrons from the tail-to-tail walls have a markedly higher kinetic energy, which can be visualized by adjusting the microscope's energy filter. ${ }^{22}$ In contrast to $\mathrm{ErMnO}_{3}$, a homogenous energy distribution is observed for the photoexcited electrons in $\mathrm{Er}_{0.99} \mathrm{Ca}_{0.01} \mathrm{MnO}_{3}$ [Fig. 1(d), gained at $643.2 \mathrm{eV}$ ]. This observation is consistent with the significantly higher bulk conductivity of Ca-doped $\mathrm{ErMnO}_{3},{ }^{19,27}$ which forbids the photoinduced surface charging, thus suppressing the formation of charging-induced domain-wall contrast at room temperature.

At low temperature, a distinctly different behavior is observed in $\mathrm{Er}_{0.99} \mathrm{Ca}_{0.01} \mathrm{MnO}_{3}$ as displayed in Fig. 2. After cooling to $160 \mathrm{~K}$, both bright and dark lines are resolved in X-PEEM [Fig. 2(a)]. The image in Fig. 2(a) is recorded after optimizing the X-ray energy to $643.1 \mathrm{eV}$ to compensate for shifts in the $\mathrm{Mn} \mathrm{L}_{2,3}$ edge so that maximum contrast is achieved. Pt-markers designed using electron-beam lithography allow
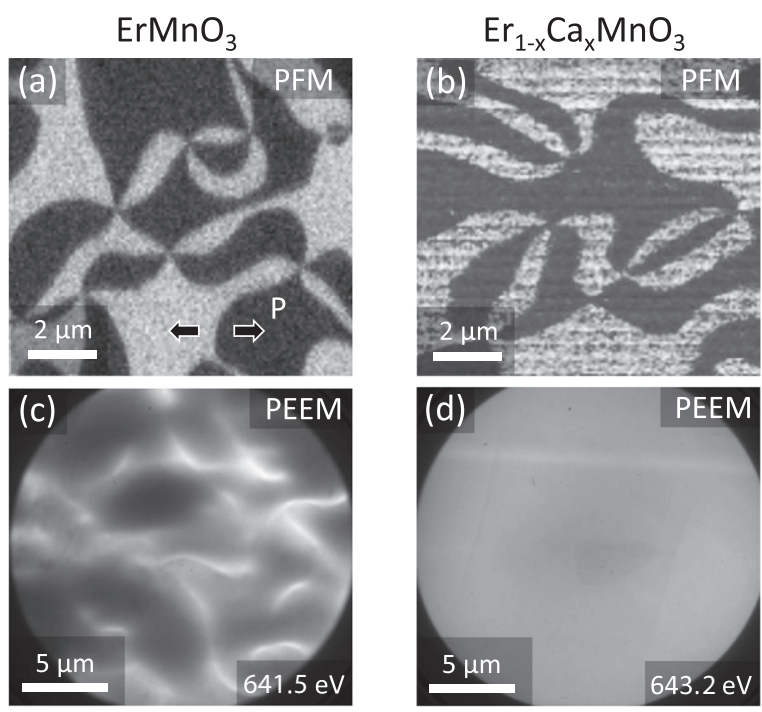

FIG. 1. (a) and (b) PFM images (in-plane contrast) of ferroelectric domains in $\mathrm{ErMnO}_{3}$ and $\mathrm{Er}_{0.99} \mathrm{Ca}_{0.01} \mathrm{MnO}_{3}$. Arrows in (a) indicate the direction of the spontaneous polarization P. (c) and (d) Room-temperature X-PEEM images at a photon energy of $641.5 \mathrm{eV}$ and $643.2 \mathrm{eV}$, respectively. ( $\mathrm{Mn} \mathrm{L}_{3}$ edge). While bright charginginduced contrast is observed at tail-to-tail domain walls in $\mathrm{ErMnO}_{3}$, a homogenous contrast level is obtained for $\mathrm{Er}_{0.99} \mathrm{Ca}_{0.01} \mathrm{MnO}_{3}$.

us to study the same sample position in subsequent cAFM and PFM scans presented in Figs. 2(b) and 2(c). The comparison with the cAFM and PFM data identifies the bright and dark lines in the lowtemperature X-PEEM image as tail-to-tail and head-to-head domain walls, respectively. Thus-different from the charging-induced contrast observed in $\mathrm{ErMnO}_{3}$ at room temperature [Fig. 1(c)] - both conducting and insulating domain walls are resolved in the low-temperature $\mathrm{X}$-PEEM images gained on $\mathrm{Er}_{0.99} \mathrm{Ca}_{0.01} \mathrm{MnO}_{3}$. This qualitative difference suggests that the low-temperature behavior is dominated by a mechanism other than photoinduced charging.

A detailed investigation of the photoelectron energy distribution at tail-to-tail and head-to-head walls $(T=160 \mathrm{~K})$ is displayed in Figs. 2(d) and 2(e). The contour-plot in Fig. 2(d) shows the normalized energy-dependent electron yield measured along the cross section marked in Fig. 2(a). The energy-dependent data are gained by detecting local intensity variations while changing the so-called start energy $U_{\text {st }}$ (the center energy of a bandpass energy filter). This allows quantification of differences in the kinetic energy of photoexcited electrons. ${ }^{22}$ Maxima of the electron distribution (light blue) are found to shift in energy at the position of the domain walls. At head-to-head walls, the electron distribution shifts to lower energies with respect to the bulk, whereas a shift to higher energies occurs at tail-to-tail walls. The latter is highlighted in Fig. 2(e), revealing an energy shift of $2.3 \pm 0.2 \mathrm{eV}$ between head-to-head and tail-to-tail walls.

Next, we consider the temperature-dependent evolution of the domain-wall signals. Figure 3 shows kinetic-energy maps obtained while heating from $160 \mathrm{~K}$ to room temperature. Maps of the spatial energy distribution are generated by fitting the secondary electron distribution for each pixel using a Gaussian fit function. At $160 \mathrm{~K}$, pronounced energy shifts are detected, which are correlated with the position of the domain walls, i.e., reduced and increased kinetic energy 
(a)

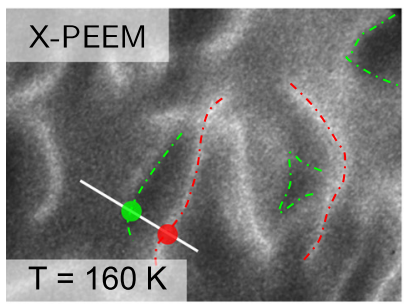

(b)

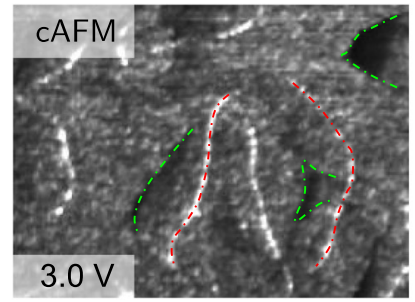

(c)

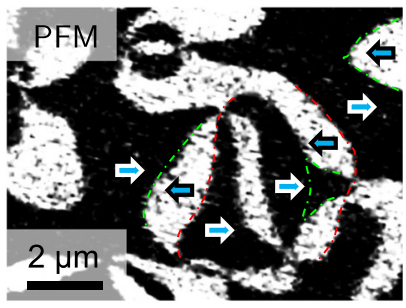

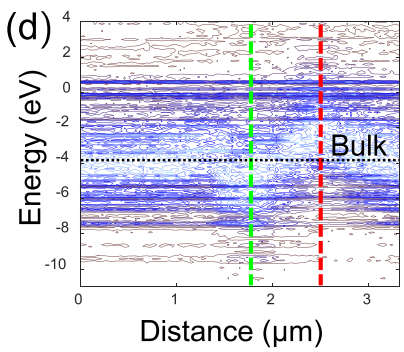

(e)

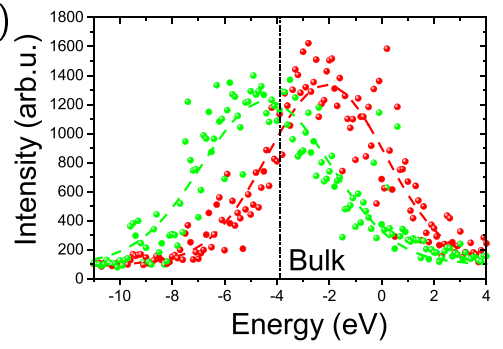

FIG. 2. (a)-(c) compare X-PEEM, CAFM, and PFM images recorded in the same area on $\mathrm{Er}_{0.99} \mathrm{Ca}_{0.01} \mathrm{MnO}_{3}$. The local conductance (cAFM) is measured at $3 \mathrm{~V}$ bias applied to the back electrode; green and red dashed lines indicate the position of insulating head-to-head (dark) and conducting tail-to-tail (bright) walls. The X-PEEM data are taken at $160 \mathrm{~K}$, whereas CAFM and PFM maps are recorded at room temperature. (d) Normalized electron yield as a function of electron energy at head-to-head (green dashed line) and tail-to-tail (red dashed line) walls measured along the cross section marked by the white line in (a) (light blue=high intensity; gray=low intensity). (e) Electron energy distribution recorded at the wall positions marked in (a) and (d). Black dashed lines in (d) and (e) correspond to the average kinetic energy of electrons emitted from the bulk.

near head-to-head and tail-to-tail domain walls, respectively. With increasing temperature, the energy contrast gradually weakens until at $181 \mathrm{~K}$ only the head-to-head walls (blue) are resolved in the X-PEEM images. This is exactly the opposite compared to X-PEEM maps gained on $\mathrm{ErMnO}_{3}$ at room temperature [Fig. 1(c) and Ref. 22], where only tail-to-tail walls are observed. This difference corroborates
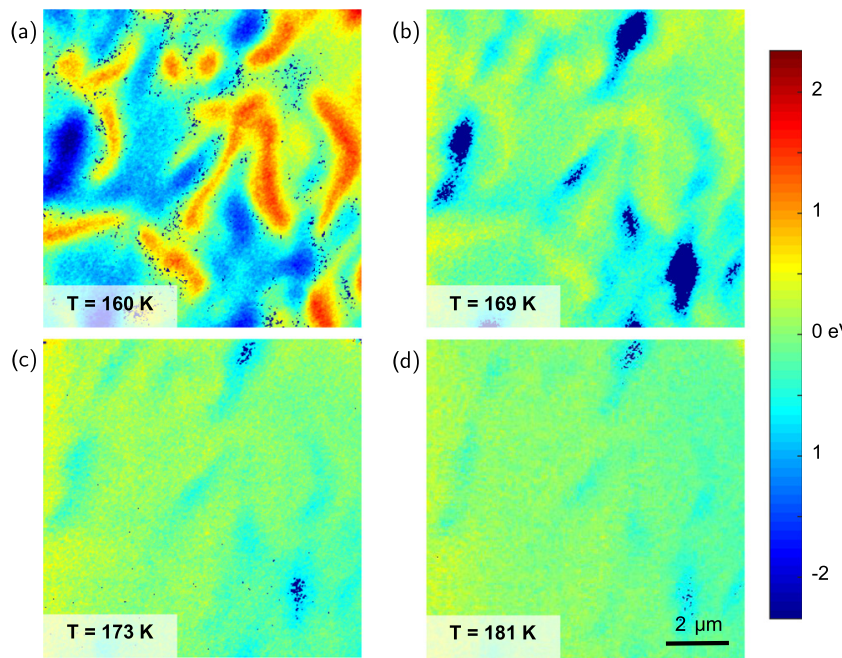

FIG. 3. (a)-(d) Temperature dependence of the spatial energy distribution of photoexcited electrons in $\mathrm{Er}_{0.99} \mathrm{Ca}_{0.01} \mathrm{MnO}_{3}$. Colors reflect the maxima of the electron energy distribution as indicated on the right. The maximum energy is derived from an energy-dependent image series, evaluating the electron energy distribution pixel-by-pixel using a Gaussian fit function. our assumption that different mechanisms are responsible for the $\mathrm{X}$-PEEM contrast at low temperature; in particular, it allows for excluding photoinduced charging as the dominant effect.

To understand the detected X-PEEM contrasts at low temperature, we consider the intrinsic properties of the system. Recent lowtemperature EFM studies revealed the emergence of uncompensated bound charges at head-to-head and tail-to-tail walls in $\mathrm{Er}_{0.99} \mathrm{Ca}_{0.01} \mathrm{MnO}_{3}$ upon cooling, ${ }^{20}$ caused by the disruption of the internal screening mechanism at low temperatures. By decreasing the temperature, the spontaneous polarization of the domains increases due to the pyroelectric effect. This further increases the bound charge at both head-to-head and tailto-tail domain walls, requiring an extra screening by mobile carriers of the bulk domains. As the effectiveness of the bulk screening is determined by mobile carriers, the screening mechanism can be disrupted due to a deficiency of mobile carriers below some characteristic temperature $T^{*}$ (note that the carrier density $n_{h}$ in the p-type semiconductor $\mathrm{Er}_{0.99} \mathrm{Ca}_{0.01} \mathrm{MnO}_{3}$ decreases exponentially, i.e., $n_{h} \propto \exp \left[-E_{F} / k T\right]$, where $k$ is the Boltzmann constant and $E_{F}$ the distance between the Fermi energy and the top of the valence band). ${ }^{20}$ After cooling to $T<T^{*}$, electrostatic equilibrium is no longer reached within the timeframe of the experiment so that extra domain-wall charges remain uncompensated, leading to pronounced electrostatic fields.

In order to test whether such uncompensated domain-wall charges can explain the low-temperature X-PEEM data, we build a model that combines previous calculations of the formation of domains $^{29}$ and domain-wall bound charges ${ }^{20}$ in hexagonal manganites, assuming the same bound charge screening behavior at head-tohead and tail-to-tail walls. For $T>T^{*}$, the material is in electrostatic equilibrium and we expect a mostly flat surface potential, which is consistent with the experimental results in Fig. 1(d). At $T<T^{*}$, however, uncompensated charges appear at the domain walls with 
maximal surface density $\sigma= \pm 2 p\left(T^{*}-T\right)$, where $p$ is the pyroelectric constant. The latter generates an electric potential $\phi(x, y)$ at the surface that shifts the kinetic energy of emitted electrons by $-e \phi(x, y)$. Figure 4 shows a Landau-theory-based simulation that illustrates the typical distribution of charged walls in hexagonal manganites ${ }^{16}$ [Fig. $4(\mathrm{a})]$ and the corresponding electric potential $\phi(x, y)$ for incomplete screening [Fig. 4(b)] [see supplementary material for details of the calculations]. White (dark) shades correspond to a lower (higher) electric potential and, therefore, to higher (lower) kinetic energies of emitted electrons. Most importantly, the calculation reproduces the main experimental feature, that is, "opposite contrast" at head-to-head and tail-to-tail walls (Figs. 3 and 4), suggesting that uncompensated bound charges do indeed play a key role in the $\mathrm{X}$-PEEM image formation at low temperature.

To show the importance of uncompensated bound charges, we make a numerical comparison of the simulated domain-wall contrast with the recorded X-PEEM data, focusing on regions with parallel domain walls. Figure 5(a) presents the relative energy shift of electrons emitted from tail-to-tail and head-to-head walls at different temperatures, corresponding to the cross section marked in the inset in Fig. 5(b). Figure 5(a) shows a pronounced asymmetry between head-to-head and tail-to-tail walls concerning the sign and magnitude of the detected energy shift, as well as its thermal evolution. The observed saw-tooth profile resembles the classic evolution of the electric potential between charged capacitor plates, and its magnitude steadily increases as the temperature decreases. In Fig. 5(b), we compare the thermal evolution of the minima (head-to-head) and maxima (tail-to-tail) in Fig. 5(a) with the temperature scaling behavior expected from the model of a plate capacitor, assuming the same maximum density of domain-wall surface charge $\sigma$ as before. Dashed lines in Fig. 5(b) are fitted to the X-PEEM data with $-e \Delta \phi= \pm e p L\left(T^{*}-T\right) / 2 \epsilon$, where $L$ is the distance between the domain walls and $\epsilon$ is the dielectric constant (we note that the data point obtained for the head-to-head wall at $160 \mathrm{~K}$ is not considered for the fit as the line plot in Fig. 5(a) indicates substantial interference with the signal associated with the tail-to-tail wall). Using $L=800 \mathrm{~nm}$ and $\epsilon=13 \epsilon_{0},{ }^{30}$ we extract the hypothetical pyroelectric constant of the material, $p=2.5 \mathrm{nC} /\left(\mathrm{cm}^{2} \mathrm{~K}\right)$. This value is in remarkable agreement with literature data for the pyroelectric constants in other manganites $\left[p\left(\mathrm{HoMnO}_{3}\right)=0.6 \mathrm{nC} /\left(\mathrm{cm}^{2} \mathrm{~K}\right) ;{ }^{31} p\left(\mathrm{YMnO}_{3}\right)=3 \mathrm{nC} /\left(\mathrm{cm}^{2} \mathrm{~K}\right)^{32}\right]$, which corroborates our bound-charge model. Furthermore, the observed
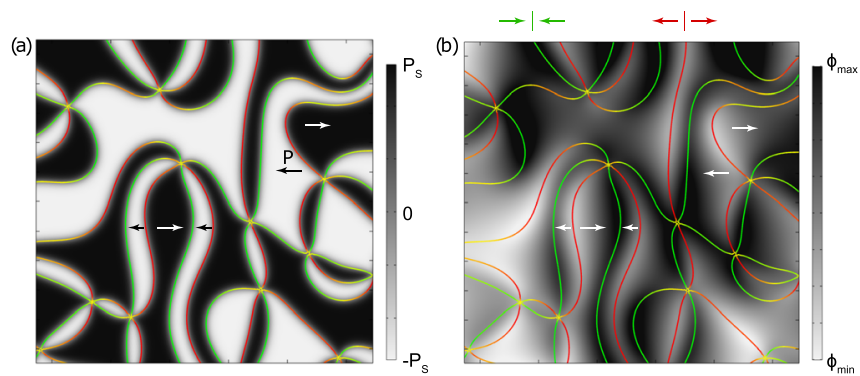

FIG. 4. (a) Calculated domain structure using the finite-element method. (b) Corresponding map of surface electric potential generated by uncompensated bound charges at head-to-head (green) and tail-to-tail (red) domain walls. Potential maxima at head-to-head walls and minima at tail-to-tail walls correspond to negative and positive eV-shifts in X-PEEM images (see Figs. 2 and 3).
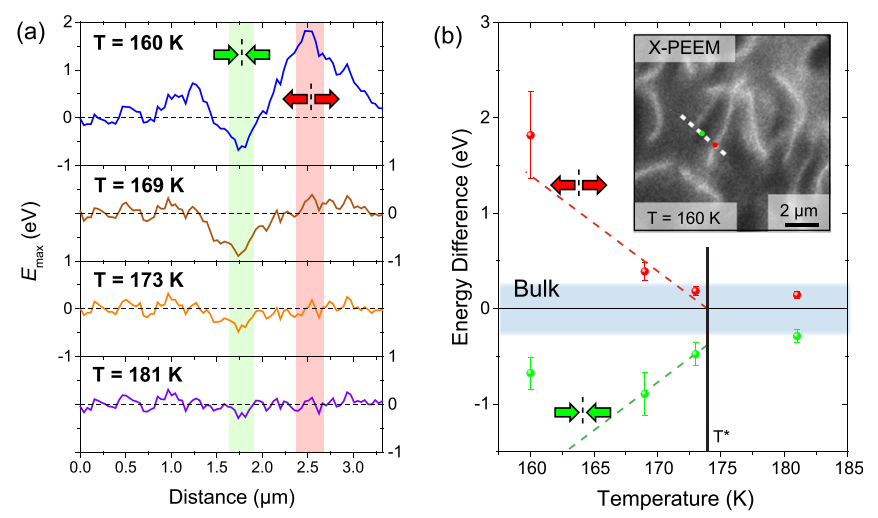

FIG. 5. (a) Energy profile of photoelectrons along the white-dashed line marked on the inset in (b). Positions of head-to-head and tail-to-tail walls are highlighted in green and red, respectively. (b) Temperature dependence of the maxima/minima in (a) relative to the bulk. Blue color indicates the uncertainty in the average energy of electrons emitted from the bulk. Dashed lines are fits based on a plate capacitor model as explained in the main text (pyroelectric constant $p=2.5 \mathrm{nC} \mathrm{cm}{ }^{-2} \mathrm{~K}^{-1}$ ).

persistence of contrast at the head-to-head domain walls toward higher temperatures compared to the tail-to-tail walls and their overall offset of the energy shift $(\approx-0.4 \mathrm{eV})$ are in qualitative agreement with the variations of the surface electric potential due to the hole depletion layer surrounding them ${ }^{9,20}$ (see supplementary material for details). Interestingly, the extracted $T^{*}=175 \mathrm{~K}$ is higher than that in previous EFM experiments $\left(T^{*}=120 \mathrm{~K}^{20}\right)$, suggesting that X-PEEM is the more sensitive technique for electrostatic potential mapping. This higher sensitivity can be partially attributed to the fact that, in contrast to the electric far fields probed in EFM (typical distance $d \approx 30 \mathrm{~nm}$ ), the much stronger electric field at the sample surface $(d \rightarrow 0)$ is responsible for image formation in X-PEEM. ${ }^{22}$ Differences in the environmental conditions are another important factor: While the EFM data in Ref. 20 have been recorded in He gas (20 mbar), X-PEEM is performed under ultrahigh vacuum $\left(6 \times 10^{-10} \mathrm{mbar}\right)$, which reduces signal loss and charge screening due to extrinsic screening by surface contamination/adsorbates. In addition, as our estimates show, surface charging and the time-dependent nature of screening disruption can contribute to higher $T^{*}$, causing a shift on the order of a few Kelvin for realistic material parameters. In conclusion, the numerical comparison of simulated and recorded X-PEEM contrasts (Fig. 5) demonstrates that the low-temperature potential maps gained on $\mathrm{Er}_{0.99} \mathrm{Ca}_{0.01} \mathrm{MnO}_{3}$ are dominated by the emergence of uncompensated domain-wall charges.

In summary, we have applied low-temperature X-PEEM to detect uncompensated bound-charges at ferroelectric head-to-head and tailto-tail domain walls in $\mathrm{Er}_{0.99} \mathrm{Ca}_{0.01} \mathrm{MnO}_{3}$. The obtained X-PEEM maps and their temperature-dependent evolution are explained based on the charge-relaxation time and the freezing of mobile carriers at low temperature. In comparison to previously applied low-temperature EFM experiments, X-PEEM readily distinguishes between positive and negative bound charges, offering high sensitivity and substantially shorter data acquisition times. This work thus introduces X-PEEM as a valuable tool for the investigation of charged domain walls and for electrostatic potential mapping in general, expanding the limited set of imaging experiments that allows for electronic property characterization at low temperature and with a nanoscale spatial resolution. 
See the supplementary material for the complete model calculations and simulation details.

We thank HZB for the allocation of synchrotron beam time, and we gratefully acknowledge financial support from HZB. J.S. and D.M. acknowledge funding from the ETH Zurich and the SNF (Proposal no. 200021_149192). D.M. thanks NTNU for support through the Onsager Fellowship Program and NTNU Stjerneprogrammet. M.S. and K.S. acknowledge the support of the European Research Council under the European Union's Horizon 2020 research and innovation program (Grant Agreement No. 724529), Ministerio de Economia, Industria y Competitividad through Grant Nos. MAT2016-77100-C2-2-P and SEV-2015-0496, and the Generalitat de Catalunya (Grant No. 2017SGR 1506). M.H. acknowledges funding from ERC (Complexplas), DFG, BW Stiftung, and MWK BW (ZAQuant, IQST). Z.Y. and E.B. were supported by the U.S. Department of Energy, Office of Science, Basic Energy Sciences, Materials Sciences and Engineering Division under Contract No. DE-AC02-05-CH11231 within the Quantum Materials Program-KC2202.

\section{REFERENCES}

${ }^{\mathbf{1}}$ E. K. H. Salje, ChemPhysChem 11, 940 (2010).

${ }^{2}$ E. K. H. Salje and H. Zhang, Phase Trans. 82, 452 (2009).

${ }^{3}$ G. Catalan, J. Seidel, R. Ramesh, and J. F. Scott, Rev. Mod. Phys. 84, 119 (2012).

${ }^{4}$ J. Seidel, L. W. Martin, Q. He, Q. Zhan, Y.-H. Chu, A. Rother, M. E. Hawkridge, P. Maksymovych, P. Yu, M. Gajek et al., Nat. Mater. 8, 229 (2009). ${ }^{5}$ J. R. Whyte, R. G. P. McQuaid, P. Sharma, C. Canalias, J. F. Scott, A. Gruverman, and J. M. Gregg, Adv. Mater. 26, 293 (2014).

${ }^{6}$ J. R. Whyte and J. M. Gregg, Nat. Commun. 6, 7361 (2015).

${ }^{7}$ D. Meier, J. Phys.: Condens. Matter 27, 463003 (2015).

${ }^{8}$ P. S. Bednyakov, B. I. Sturman, T. Sluka, A. K. Tagantsev, and P. V. Yudin, npj Comp. Mater. 4, 65 (2018).

${ }^{9}$ J. A. Mundy, J. Schaab, Y. Kumagai, A. Cano, M. Stengel, I. P. Krug, D. M. Gottlob, H. Doğanay, M. E. Holtz, R. Held et al., Nat. Mater. 16, 622 (2017).

${ }^{10}$ J. Schaab, S. H. Skjærvø, S. Krohns, X. Dai, M. E. Holtz, A. Cano, M. Lilienblum, Z. Yan, E. Bourret, D. A. Muller et al., Nat. Nanotechnol. 13, 1028 (2018).
${ }^{17}$ V. V. Aristov, L. S. Kokhanchik, and Y. I. Voronovskii, Phys. Status Solidi A 86, 133 (1984).

${ }^{12}$ J. Li, H. X. Yang, H. F. Tian, C. Ma, S. Zhang, Y. G. Zhao, and J. Q. Li, Appl. Phys. Lett. 100, 152903 (2012).

${ }^{13}$ T. Sluka, A. K. Tagantsev, D. Damjanovic, M. Gureev, and N. Setter, Nat. Commun. 3, 748 (2012).

${ }^{14}$ J. Guyonnet, I. Gaponenko, S. Gariglio, and P. Paruch, Adv. Mater. 23, 5377 (2011).

${ }^{15}$ S. Farokhipoor and B. Noheda, Phys. Rev. Lett. 107, 127601 (2011).

${ }^{16}$ D. Meier, J. Seidel, A. Cano, K. Delaney, Y. Kumagai, M. Mostovoy, N. A. Spaldin, R. Ramesh, and M. Fiebig, Nat. Mater. 11, 284 (2012).

${ }^{17}$ P. Maksymovych, A. N. Morozovska, P. Yu, E. A. Eliseev, Y.-H. Chu, R. Ramesh, A. P. Baddorf, and S. V. Kalinin, Nano Lett. 12, 209 (2012).

${ }^{18}$ M. Schröder, A. Haußmann, A. Thiessen, E. Soergel, T. Woike, and L. M. Eng, Adv. Funct. Mater. 22, 3936 (2012).

${ }^{19}$ J. Schaab, A. Cano, M. Lilienblum, Z. Yan, E. Bourret, R. Ramesh, M. Fiebig, and D. Meier, Adv. Electron. Mater. 2, 1500195 (2016).

${ }^{20}$ P. Schoenherr, K. Shapovalov, J. Schaab, Z. Yan, E. D. Bourret, M. Hentschel, M. Stengel, M. Fiebig, A. Cano, and D. Meier, Nano Lett. 19, 1659 (2019).

${ }^{21}$ Y. Geng, N. Lee, N. Y. J. Choi, S.-W. Cheong, and W. Wu, Nano Lett. 12, 6055 (2012).

${ }^{22}$ J. Schaab, I. P. Krug, F. Nickel, D. M. Gottlob, H. Doğanay, A. Cano, M. Hentschel, Z. Yan, E. Bourret, C. M. Schneider et al., Appl. Phys. Lett. 104, 232904 (2014).

${ }^{23}$ A.-S. Pawlik, T. Kämpfe, A. Haußmann, T. Woike, U. Treske, M. Knupfer, B. Büchner, E. Soergel, R. Streubel, A. Koitzsch et al., Nanoscale 9, 10933 (2017).

${ }^{24}$ Z. Zhao, N. Barrett, Q. Wu, D. Martinotti, L. Tortech, R. Haumont, M. Pellen, and E. K. H. Salje, Phys. Rev. Mater. 3, 043601 (2019).

${ }^{25}$ I. Stolichnov, L. Feigl, L. J. McGilly, T. Sluka, X.-K. Wie, E. Colla, A. Crassous, K. Shapovalov, P. Yudin, A. K. Tagantsev et al., Nano Lett. 15, 8049-8055 (2015).

${ }^{26}$ E. Y. Ma, Y.-T. Cui, K. Ueda, S. Tang, K. Chen, N. Tamura, P. M. Wu, J. Fujioka, Y. Tokura, and Z.-X. Shen, Science 350, 538 (2015).

${ }^{27}$ E. Hassanpour, V. Wegmayr, J. Schaab, Z. Yan, E. Bourret, Th. Lottermoser, M. Fiebig, and D. Meier, New. J. Phys. 18, 43015 (2016).

${ }^{28}$ Z. Yan, D. Meier, J. Schaab, R. Ramesh, E. Samulon, and E. Bourret, J. Cryst. Growth 409, 75 (2015).

${ }^{29}$ M. Holtz, K. Shapovalov, J. A. Mundy, C. S. Chang, Z. Yan, E. Bourret, D. A. Muller, D. Meier, and A. Cano, Nano Lett. 17, 5883 (2017).

${ }^{30}$ M. Stengel, C. J. Fennie, and P. Ghosez, Phys. Rev. B 86, 094112 (2012).

${ }^{31}$ N. Hur, I. K. Jeong, M. F. Hundley, S. B. Kim, and S.-W. Cheong, Phys. Rev. B 79, 134120 (2009).

${ }^{32}$ S. Artyukhin, K. Delaney, N. A. Spaldin, and M. Mostovoy, Nat. Mater. 13, 42 (2014). 